\title{
VIBRATION ATTENUATION OF BORING BARS WITH NONLINEAR CONTROL FORCE
}

\author{
Z. Iklodi ${ }^{1}$, A. Astarloa ${ }^{2}$, Z. Dombovari ${ }^{1,2 *}$ \\ ${ }^{1}$ Budapest University of Technology and Economics, Department of Applied Mechanics, Budapest, Hungary \\ ${ }^{2}$ IK4-Ideko, Dynamics \& Control, Elgoibar, Basque Country, Spain \\ *Corresponding author; e-mail: dombovari@mm.bme.hu
}

\begin{abstract}
An active method for boring bar vibration attenuation and chatter suppression is presented through the introduction of a nonlinear control force within a tuned mass damper. Its effects on process stability and its control options are discussed in detail. The effectiveness of the active damping system and the optimal control parameters are determined through numerical simulations carried out on a simplified linearized mechatronic model of the coupled nonlinear manufacturing system.
\end{abstract}

\section{Keywords:}

Boring bar; Active damper; Nonlinear force; Linear stability

\section{INTRODUCTION}

The boring machining process is highly restricted due to stability boundaries in connection with the geometry of the bores and the dynamical properties of the machine tool. The production of long, slim bores is most difficult since it requires a slender boring bar with highly inconvenient mechanical properties. Due to small machine tool stiffness, the appearance of regenerative chatter is a common difficulty, causing poor surface finish and extensive tool wear. The biggest and most important engineering challenge of boring is to extend the limit of the chatter-free tool length to diameter $(L / D)$ ratio, therefore expanding the spectrum of achievable bore geometries.

Major efforts have been made already in the field of machine tool vibration attenuation and chatter suppression. There are several active and passive methods available, discussed in further detail in the works of Munoa et al. [Munoa 2016]. In the case of the boring, passive vibration attenuation methods often fall short in the face of increasing industrial needs and requirements.

In order to expand the chatter-free $L / D$ ratio above the passively achievable limit, several different active damping techniques have been developed. Such as the ones mentioned in the works of Åkesson et al. [Åkesson 2006] and Mei et al. [Mei 2005]. Most of these solutions using some sort of feedback control based on position, velocity or chatter frequency, and a force actuator either magnetic, piezoelectric or hydraulic.

As presented in the works of Tanaka et al. [Tanaka 1994] and Redmond et al. [Remond 1999], piezoelectricity is an efficient approach for active boring bar vibration attenuation, through the generation of internal bending moments. They propose chatter frequency and displacement, velocity, acceleration based control strategies to provide the necessary active damping. In the former study the optimal position of the actuator is also determined.

Piezoelectric actuators can also be used for active error correction in high precision boring. Such a method is presented by Chiu et al. [Chiu 1997] via a PC controlled active piezoelectric tool holder. Actuation is done based on displacement measured trough strain gauges and a PID control loop.

Magnetic actuators can also be used for active vibration attenuation. Either through external non-contact clamping [Chen 2014], or via an internal actuated inertial mass [Abele 2016]. Both studies suggest the use of at counteracting pairs of current controlled electromagnets, and the use of permanent magnets to amplify actuation forces. As feedback variable, relative displacement is used and with Abele et al. [Abele 2016] it is aided by acceleration data. Both of these can be easily measured with fiber optics, and piezo accelerometers.

Another approach to boring bar vibration attenuation is the use of passive, semi active or active tuned mass dampers (TMD's). This topic is further discussed in [Munoa 2015], where the effect of TMD's - with passive Den Hartog [Den Hartog 1934] and Sims [Sims 2007] tuning, and semi active self-tuning - on the stability of a milling process is investigated. Regarding to the robustness of the process and the achievable material removal rates, it states, that the use of TMD's is beneficial overall, and the use of an active damping system can improve the machining process even further. In accordance with these results, the introducing of an actively controlled and actuated TMD to the boring process proposes favorable results.

In this current paper a novel method for active vibration attenuation is presented through the introduction of a nonlinear control force in the applied tuned mass damper. The proposed method as seen in Fig. 1, is an active vibration attenuation system with a broad range of control 
options. Given the right control strategy this approach can greatly improve the quality and the productivity of the boring machining process, and can help increase the limit of chatter free operable boring bar $L / D$ ratio.

The study aims to propose an active control method for slender boring bars with $L / D$ ratio of $7-8$, with the focus on providing a generic control strategy independent of physical realization. Thus in all calculations arbitrarily chosen sample data was used, and the method should be applicable to a wide range of boring bar sizes.

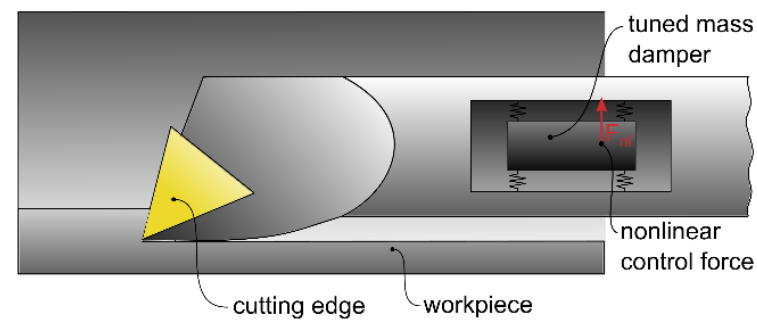

Fig. 1: Nonlinear active damper.

The introduction of the nonlinear control force despite providing active control options also causes the machining system to become unstable on its own. Consequently finding the optimal control parameters for stable operation is of the outmost importance.

In order to achieve the above described feat an adequately accurate model of the system must be created. First the nonlinear force characteristics should be investigated trough linearization, then it should be combined with the regenerative force model of the orthogonal cutting process. Accomplishing that, the resulting mathematical model was a highly complex system of nonlinear delayed differential equations, therefore in order to perform further tests, simplifications and linearization were needed.

With the help of the linearized model of the machining system, the ideal control strategy was determined. Taking the measurability and controllability of the state variables into account, a PDA12 voltage control was introduced, since the control force was assumed to be actuator voltage dependent. As feedback, relative positions and velocities, and absolute accelerations - of the damper and the tool were used. Acceleration was chosen as a control variable with the intent of introducing virtual masses to the system, therefore battling the most significant limiting factor of TMD's, the maximal achievable weight ratio.

The stable control parameters were determined using a stability analysis based on a Linear Multistep Method (LMS, [Engelborghs 2002]) on the linearized form of the mechatronic model. Then the performance of the active damping system was compared to its ideally tuned passive counterparts.

\section{MODELLING}

The presented mechanical model was greatly simplified, excluding continuum mechanical problems, with replacing the entirety of the boring bar with a concentrated mass a spring and a damping, at the point of cutting.

For modelling the effects of cutting, a linear force model was used, thus providing a conveniently simple mathematical representation, and an analytical solution to compare the results to.

\subsection{Control force}

The aim of this current study was to enhance the performance of passive boring bar vibration attenuation systems with the introduction of a nonlinear control force. Such nonlinear force characteristics are often present in active systems, due to the inclusion of force actuators, such as magnetic, piezoelectric and hydraulic actuators.

For the nonlinear control force, the following arbitrary characteristics was chosen:

$F_{\mathrm{nl}}=K\left(\frac{U_{1}^{2}}{(a-x)^{n}}-\frac{U_{2}{ }^{2}}{(a+x)^{n}}\right)$.

This formula represents the combined effect of two actuators, where the actuating force is proportional to the square of the applied voltage, and is in inverse relationship with the $\mathrm{n}^{\text {th }}$ power of the position of the actuated mass $x . K$ represents a force coefficient, $U_{1}$ and $U_{2}$ denotes the applied actuator voltages, and $a$ is the equilibrium distance, where given the same excitation the effect of the actuators cancel each other out.

This resultant force is highly nonlinear, and it diverges to infinity as the actuated mass collides into the actuator. It also makes the equilibrium at $x=0$, unstable since here its derivative by $x$ is always positive. All these mentioned above are rather inconvenient properties, thus without a proper control strategy, the introduction of the nonlinear control force is actually harmful for the operation of the boring process.

\subsection{Nonlinear active damper}

Designing a nonlinear active damper requires a sufficiently accurate yet simple model of the coupled machining system. Such a model can be constructed by adding a position dependent force source representing the nonlinaear actuators to the passive vibration damper, as seen in Fig. 2. The actuated force $F_{\mathrm{nl}}$, can be calculated here using the formula seen in equation (2). In Fig. 2. $m_{1}, k_{1}$ and $b_{1}$ represent the modal mass, stiffness and damping of the machine tool, while $m_{2}, k_{2}$ and $b_{2}$ denote the mass, stiffness and damping of the TMD. The PC represents the control system which uses the positions, velocities and accelerations of the machine tool and TMD: $x 1, x 2$ and their derivatives as feedback variables, to provide the necessary voltages $U_{1}$ and $U_{2}$ for the included actuator. The working principles of the control system and the measurement of feedback variables is discussed in further detail in section 3.

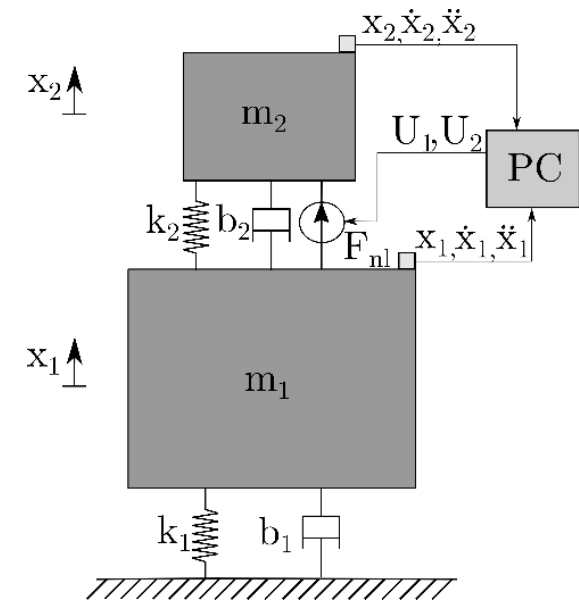

Fig. 2: Modelling of active dampers considering feasible sensor types and positions. 
$F_{\mathrm{nl}}=K\left(\frac{U_{1}^{2}}{\left(a-x_{2}+x_{1}\right)^{2}}-\frac{U_{2}^{2}}{\left(a-x_{2}+x_{1}\right)^{2}}\right)$.

For further analysis and control design the linearization of the active control force is needed. It can be done around the $x_{1}=x_{2}=0$ equilibrium, assuming that $U_{1}=U_{2}$ and the springs are tensionless in this state (3).

$F_{\mathrm{nl}} \approx F_{\mathrm{nl}, 0}+\left.\frac{\partial F_{\mathrm{nl}}}{\partial x_{1}}\right|_{x_{1,2}=0} x_{1}+\left.\frac{\partial F_{\mathrm{nl}}}{\partial x_{2}}\right|_{x_{1,2}=0} x_{2}=-\Psi x_{1}+\Psi x_{2} \cdot$ (3)

Where $\psi$ is a positive constant:

$\Psi:=\frac{K n}{a^{n+1}}\left(U_{1}^{2}+U_{2}^{2}\right)$.

Using equation (2) a linearized equation of motion can be formulated for the simplified two degree of freedom active damper system (5).

$\mathbf{M} \ddot{\mathbf{x}}+\mathbf{C} \dot{\mathbf{x}}+\mathbf{K} \mathbf{x}=\mathbf{0}$,

where:

$\mathbf{M}=\left(\begin{array}{cc}m_{1} & 0 \\ 0 & m_{2}\end{array}\right)$,

$\mathbf{C}=\left(\begin{array}{cc}b_{1}+b_{2} & -b_{2} \\ -b_{2} & b_{2}\end{array}\right)$,

$\mathbf{K}=\left(\begin{array}{cc}k_{1}+k_{2}-\Psi & \Psi-k_{2} \\ \Psi-k_{2} & k_{2}-\Psi\end{array}\right)$.

From equation (6.3) it is visible, that if $\psi$ is greater than $k_{2}$, meaning the nonlinear active forces overpower the spring forces, the stiffness matrix loses its positive definiteness, thus causing the solution of the equations to become unstable.

\subsection{Cutting process}

Since the main focus of this current study was active damping system development not boring bar design, al problems related to the tool geometry were disregarded. Through the modelling process, the boring bar was replaced with a concentrated mass, a spring and a viscose damping. These components are meant to model the bending dynamics of the boring bar at the point of cutting. The simplified representation of a turning process executed by an active TMD assisted boring bar can be seen in Fig. 3 .

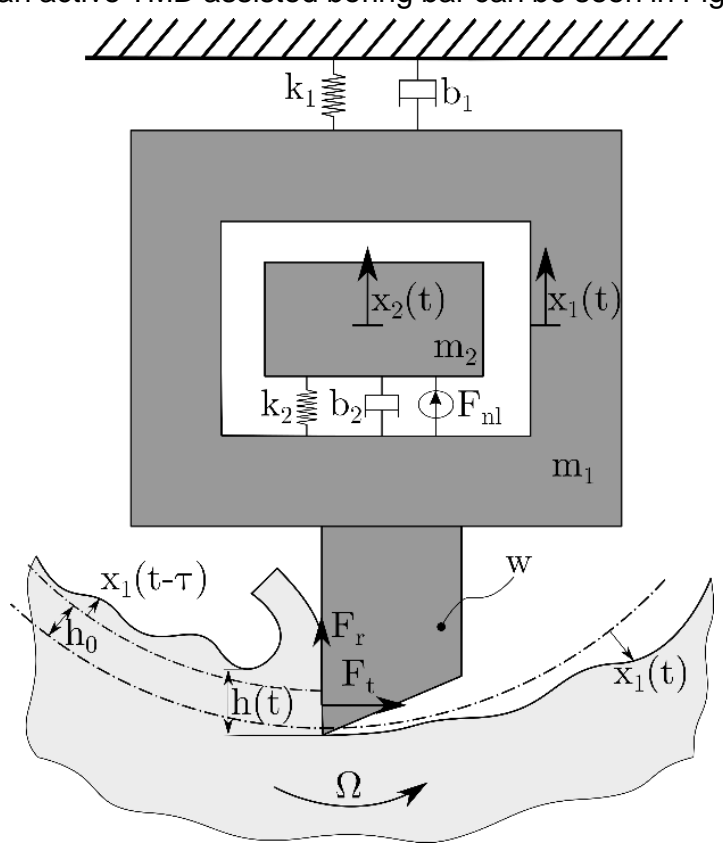

Fig. 3: Simplified boring bar model.
Here the notations are the same as on Fig. 2., with the addition of $h_{0}$ : desired depth of cut, $h$ : actual depth of cut, $F_{t}$ and $F_{r}$ : tangential and radial cutting force, $w$ : feed rate and $\Omega$ : spindle speed.

In order to keep the mathematical model of the system as simple as possible, a linear regenerative force model [Marui 1988] was used to represent the effects of the turning operation. Since the inclusion of the damper in the boring bar is meant to attenuate bending vibrations, it is enough to describe the behavior of the system only along one dimension with 2 DOF. Thus only the $x$ directional component of the cutting force was taken into account, which can be calculated using equation (7).

$F_{C x}(t)=\chi w\left(K_{\mathrm{e} t}+K_{\mathrm{c} t}\left(h_{0}+x_{1}(t-\tau)-x_{1}(t)\right)\right)$.

Where $X, K_{\mathrm{e} t}$ and $K_{\mathrm{c} t}$ are empirical force constants that can be obtained from orthogonal cutting tests. $w$ denotes the feed rate, $h_{0}$ the desired depth of cut and $x_{1}$ the position of the cutting edge. This force model takes the regenerative nature of the process into account, introducing a delayed component into the mathematical description of the system, representing the trace the cutting edge left on the workpiece throughout its previous revolution. Consequently the introduced time delay is equal to the period of one rotation, and can be calculated from the angular velocity (a.k.a. spindle speed, $\Omega$ ) used for the cutting process (8).

$\tau=\frac{2 \pi}{\Omega}$.

Incorporating the effects of the cutting force into the previously defined mathematical model of the 2DOF nonlinear active damper (5), the equation of motion describing the attenuated boring process can be derived. Since a linear cutting force model was used, and the constant parts of the cutting force can be cancelled out by spring preload, which will have no significant effect on the dynamic behavior, no further linearization is needed. Thus the following linear equation of motion was derived:

$\mathbf{M} \ddot{\mathbf{x}}(t)+\mathbf{C} \dot{\mathbf{x}}(t)+\left(\mathbf{K}+\mathbf{F}_{\mathrm{C}}\right) \mathbf{x}(t)=\mathbf{F}_{\mathrm{C} \tau} \mathbf{x}(t-\tau)$.

Where:

$\mathbf{F}_{\mathrm{C}}=\mathbf{F}_{\mathrm{C} \tau}=\left(\begin{array}{cc}\chi w K_{c t} & 0 \\ 0 & 0\end{array}\right)$.

\section{CONTROL DESIGN}

As it has been shown previously, the introduction of the nonlinear control force is indeed harmful without proper control. Thus the development of a viable control strategy is of the outmost importance.

At first the measurability and controllability of the state variables was investigated. Direct voltage control was used, since the nonlinear force was assumed to be voltage dependent, as seen in the formula in equation (1). This current study does not go into detail regarding the in practice execution of the voltage regulation, and assumes instantaneous and arbitrary changes can be executed by the power electronics.

Relative position, relative velocity and absolute acceleration were chosen as feedback variables, due to measurability. Accelerations can be easily evaluated individually via piezoelectric sensors, distance between the damper mass and the boring bar can be measured using laser displacement sensors, and for the relative velocity laser surface velocimetry is a viable option. However, it is rather difficult to measure velocity or displacement individually for both bodies. 
Considering all of the above a PDA12 voltage control was chosen. In the presence of this control strategy, the nonlinear actuator voltages can be calculated as seen in equation (11).

$$
\begin{aligned}
& U_{1}=U_{10}-P\left(x_{2}-x_{1}\right)-D\left(\dot{x}_{2}-\dot{x}_{1}\right)+A_{1} \ddot{x}_{1}-A_{2} \ddot{x}_{2}, \\
& U_{2}=U_{20}+P\left(x_{2}-x_{1}\right)+D\left(\dot{x}_{2}-\dot{x}_{1}\right)-A_{1} \ddot{x}_{1}+A_{2} \ddot{x}_{2} .
\end{aligned}
$$

Since as seen in equation (1) the active nonlinear force and the applied voltages are in quadratic relation, the repetition of the linearization step seen in equation (3) is necessary. However this time the control force will also depend on the acceleration and velocity values of the masses. If we assume that in equilibrium $U_{10}=U_{20}=U_{0}$, than the linearization will yield the following formula:

$$
\begin{aligned}
& F_{m} \approx F_{m 0}+\left.\frac{\partial F_{m}}{\partial x_{1}}\right|_{\substack{\dot{x}_{1,2}=0 \\
\dot{1}_{1,2}=0}} x_{1}+\left.\frac{\partial F_{m}}{\partial x_{2}}\right|_{\substack{x_{1,2}=0 \\
\dot{x}_{1,2}=0}} x_{2}+\left.\frac{\partial F_{m}}{\partial \dot{x}_{1}}\right|_{\substack{1,2 \\
\dot{x}_{1,2}=0}} \dot{x}_{1}+ \\
& \left.\frac{\partial F_{m}}{\partial \dot{x}_{2}}\right|_{\substack{1,2=0 \\
\dot{x}_{1,2}=0}} \dot{x}_{2}+\left.\frac{\partial F_{m}}{\partial \ddot{x}_{1}}\right|_{\substack{x_{1,2}=0 \\
\dot{x}_{1,2}=0}} \ddot{x}_{1}+\left.\frac{\partial F_{m}}{\partial \ddot{x}_{2}}\right|_{\substack{\ddot{x}_{1,2}=0 \\
\dot{x}_{1,2}=0}} \ddot{x}_{2}=(P \Gamma-\Psi) \mathrm{x}_{1}- \\
& \ddot{x}_{1,2}=0 \quad \ddot{x}_{1,2}=0 \quad \ddot{x}_{1,2}=0 \\
& (P \Gamma-\Psi) \mathrm{x}_{2}+D \Gamma \dot{x}_{1}-D \Gamma \dot{x}_{2}+A_{1} \Gamma \ddot{x}_{1}-A_{2} \Gamma \ddot{x}_{2},
\end{aligned}
$$

where $\psi$ and $\Gamma$ are positive constants:

$\Psi:=\frac{2 K n}{a^{n+1}} U_{0}^{2}$,

$\Gamma:=\frac{4 K}{a^{n+1}} U_{0}$.

Using the new linearized form of the active nonlinear force, the linear equation of motion for the actively controlled vibration attenuation supported boring process can be derived (14).

$\mathbf{M} \ddot{\mathbf{x}}(t)+\mathbf{C} \dot{\mathbf{x}}(t)+\left(\mathbf{K}+\mathbf{F}_{\mathrm{C}}\right) \mathbf{x}(t)=\mathbf{F}_{\mathrm{C} \tau} \mathbf{x}(t-\tau)$.

Where:

$$
\begin{aligned}
& \mathbf{M}=\left(\begin{array}{cc}
m_{1}+A_{1} \Gamma & -A_{2} \Gamma \\
-A_{1} \Gamma & m_{2}+A_{2} \Gamma
\end{array}\right), \\
& \mathbf{C}=\left(\begin{array}{cc}
b_{1}+b_{2}+D \Gamma & -b_{2}-D \Gamma \\
-b_{2}-D \Gamma & b_{2}+D \Gamma
\end{array}\right), \\
& \mathbf{K}=\left(\begin{array}{cc}
k_{1}+k_{2}-\Psi+P \Gamma & \Psi-k_{2}-P \Gamma \\
\Psi-k_{2}-P \Gamma & k_{2}-\Psi-P \Gamma
\end{array}\right) .
\end{aligned}
$$

\section{SIMULATIONS}

With the help of the created simplified machining model of the actively controlled vibration attenuated boring process, numerical tests can be carried out to compare the effectiveness of the novel active damper, to its already in practice passive counterparts.

Since the equation of motion derived in equation (14) is a delay differential equation, in general sense no analytical solution can be found, and a numerical method must be chosen for its examination. In this current study the AdamsBashford linear approximation formula, a version of Linear Multistep (LMS) methods was used [Engelborghs 2002].

To apply an LMS method, the equation of motion seen in (14) must be rewritten in the generic form of a first order DDE (16).

$\dot{\mathbf{y}}(t)=\mathbf{L} \mathbf{y}(t)+\mathbf{R} \mathbf{y}(t-\tau)$,

where:

$\mathbf{y}=\left(\begin{array}{llll}x_{1} & x_{2} & \dot{x}_{1} & \dot{x}_{2}\end{array}\right)^{\top}$,

$\mathbf{L}=\left(\begin{array}{cc}\mathbf{0} & \mathbf{I} \\ -\mathbf{M}^{-1}\left(\mathbf{K}+\mathbf{F}_{\mathrm{C}}\right) & -\mathbf{M}^{-1} \mathbf{C}\end{array}\right)$
$\mathbf{R}=\left(\begin{array}{cc}\mathbf{0} & \mathbf{0} \\ -\mathbf{M}^{-1} \mathbf{F}_{\mathrm{R} \tau} & \mathbf{0}\end{array}\right)$

Applying the Adams-Bashford method [Engelborghs 2002] to equation (16) results in the following linear algebraic forward stepping formula capable of approximating the solution of the delay differential equation:

$\mathbf{y}_{s+k}=\mathbf{y}_{s+k-1}+h \sum_{j=0}^{k-1} \beta_{j}\left(\mathbf{L y}_{s+j}+\mathbf{R} \mathbf{y}_{S+j-N}\right)$.

In equation (18) $h$ is the time step used for the approximation, which was chosen so $h=T / N$, thus no interpolation is needed upon enumerating the delayed term. The $\beta_{j}$ approximation constants can be calculated using the following formula [Iserles 1996]:

$\beta_{j}=\frac{(-1)^{j}}{j !(k-j-1) !} \int_{0}^{1} \prod_{\substack{i=0 \\ i \neq j}}^{k-1}(u+i) d u, \quad \mathrm{j}=0, \ldots, \mathrm{k}-1$.

With the help of the LMS method, the stability of the solution of the original linear equation of motion (14), can be easily investigated by formulating a linear operation $\boldsymbol{z}_{s+1}=\mathbf{S} \mathbf{z}_{s}$ from equation (18). This linear operation can be represented by a stepmatrix seen in the following equation:

$$
\mathbf{S} \in \mathbb{R}^{(N+k) \times(N+k)}=
$$$$
\left(\begin{array}{cccccccccc}
-\mathbf{I}+h * \beta_{k-1} \mathbf{L} & h \beta_{k-2} \mathbf{L} & \ldots & h \beta_{0} \mathbf{L} & \mathbf{0} & \ldots & \mathbf{0} & h \beta_{k-1} \mathbf{R} & \ldots & h \beta_{0} \mathbf{R} \\
\mathbf{I} & \mathbf{0} & \ldots & \ldots & \ldots & \ldots & \ldots & \ldots & \ldots & \mathbf{0} \\
\mathbf{0} & \mathbf{I} & \ldots & \ldots & \ldots & \ldots & \ldots & \ldots & \ldots & \mathbf{0} \\
\vdots & \vdots & \ddots & \vdots & \vdots & \vdots & \vdots & \vdots & \vdots & \vdots \\
\mathbf{0} & \ldots & \ldots & \ldots & \ldots & \ldots & \ldots & \ldots & \mathbf{I} & \mathbf{0}
\end{array}\right) .
$$

Through the eigenvalues of the stepmatrix $\mathbf{S}$ seen in equation (20), the stability of the linear operation along with the stability of the original delayed differential equation system can be investigated. If all eigenvalues have an absolute value less than 1 , than the solution of the equation of the motion and therefore the boring process will remain stable.

It is clear, that the LMS method is capable of determining the stability of the DDE describing the active controlled boring process, however before carrying out actual numerical simulations its accuracy must be tested. A simple one degree of freedom turning process is ideal for this application since it has an analytical solution to which the numerical results can be compared to. Such a comparison can be seen in Fig. 4.

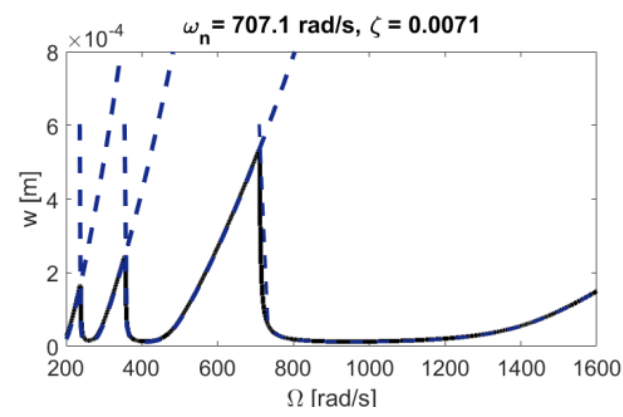

Fig. 4: Analytical and numerical solution comparison.

As seen on the $w-\Omega$ stability maps on Fig. 4 , the LMS numerical method provides a valid approach for estimating stability maps of turning operations. Furthermore its accuracy, convergence and correlation with the original DDE is proven by Engelborghs et al. in their corresponding research [Engelborghs 2002].

It is worth mentioning, that the accuracy of the AdamsBashforth method is highly dependent on the time step used. This is especially problematic upon investigating the stability of milling processes on low spindle speeds. As the angular velocity of the part decreases, the time delay $T$ 
increases, and in order to keep the used time step $h$ on a sufficiently small value, the step matrix dimension $N$ has to be increased. However this leads to a significant increase in the required computational power. In order to keep computing needs low, the simulations carried out were focused on higher angular velocity levels.

\section{RESULTS}

The goal of this current study was to develop an active control system for boring bars, which can improve the efficiency and productivity of the machining process. In order to do so, during the simulations, the nonlinear control force was added to an already ideally tuned passive damper, to see whether their inclusion can yield any benefits.

Since the goal was to create a generic control strategy independent of physical realization, throughout the testing and tuning of the active control, plausible example data was used to carry out the numeric simulations. The parameters of the applied passive damper were tuned according to Sims' formulas [Sims 2007]. All these above mentioned data can be found in Tab 1.

Tab. 1: Example data.

\begin{tabular}{cccc}
\hline sgn. & description & value & unit \\
\hline$m_{1}$ & Weight of machine tool & 1 & $\mathrm{~kg}$ \\
$k_{1}$ & Stiffness of machine tool & $5 \mathrm{e} 5$ & $\mathrm{~N} / \mathrm{m}$ \\
$b_{1}$ & Damping of machine tool & 10 & $\mathrm{Ns} / \mathrm{m}$ \\
$m_{2}$ & Damper mass & 0.05 & $\mathrm{~kg}$ \\
$k_{2}$ & Damper stiffness & $2.69 \mathrm{e} 4$ & $\mathrm{~N} / \mathrm{m}$ \\
$b_{2}$ & Damper damping & 9.45 & $\mathrm{Ns} / \mathrm{m}$ \\
$K_{c t}$ & Cutting edge coefficient & $2 \mathrm{e} 9$ & $\mathrm{~Pa}$ \\
$X$ & Force ratio & 0.3 & 1 \\
$n$ & Nonlinear exponent & 2 & 1 \\
$a$ & Equilibrium distance & 5 & $\mathrm{~mm}$ \\
$K$ & Force coefficient & $7.88 \mathrm{e}-10$ & $\mathrm{Nm}{ }^{2} /$ \\
$U_{0}$ & Equilibrium voltage & 20 & $\mathrm{~V}$ \\
\hline
\end{tabular}

In order to improve the effectiveness of the boring process with the inclusion of active control, first the initial performance of the passive damper system must be examined. The cutting speed - feed rate stability maps of the boring process in the presence of ideally tuned passive dampers can be seen in Fig. 5 In this application Sims tuning is more favourable compared to the Den Hartog one [Den Hartog 1934], since the minimum of the lobes is higher, and it provides safer operation in the whole cutting speed scale.

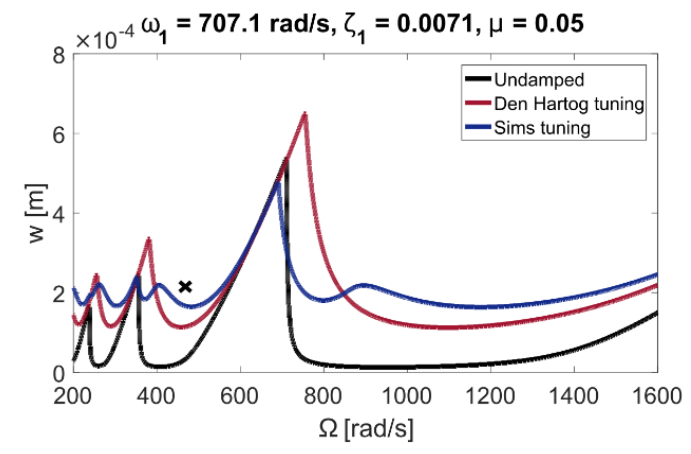

Fig. 5: Boring stability map in presence of passive dampers.
Consequently the goal of control strategy development was to find control parameters capable of expanding the Sims $w-\Omega$ stability map. Thus as seen in Fig. 5, a passively unstable parameter combination was chosen as the focus of further simulations, since if this feed rate - cutting speed combination can be operated safely with the help of active control, then the introduction of the nonlinear control force will prove itself beneficial.

\subsection{PD approach}

Given the example data seen in Tab. 1, without active control, the introduction of the nonlinear control force causes the system to become initially unstable, even without taking the regenerative effects of cutting into account. However this effect can be counterbalanced using simple PD voltage control on the actuators.

For successful active control adequate PD parameters must be found, in presence of which, the boring process remains stable. Since the goal was to improve the passive vibration attenuation strategy, the unstable $w-\Omega$ combination shown on Fig. 6 was used throughout the search of control parameters.

The result of this search can be seen on the P-D stability map seen on Fig. 6. Here it is clear that stable parameters can be found for one passively unstable point of operation. however for testing the efficiency of the active control the whole $w-\Omega$ map must be examined in its presence. An arbitrarily chosen example PD combination was selected and tested in this manner, resulting in Fig 8. From this graph it is visible, that previously unstable feed rate - cutting speed combinations become stable thanks to the active enhancement, and higher material removal rates become available.

In the presence of the active control, the minimum of the lobes decreased, and examining the whole cutting speed scale, the cutting process became less robust. The control parameters however can be tuned in accordance with the used spindle speed, thus dismissing this drawback.

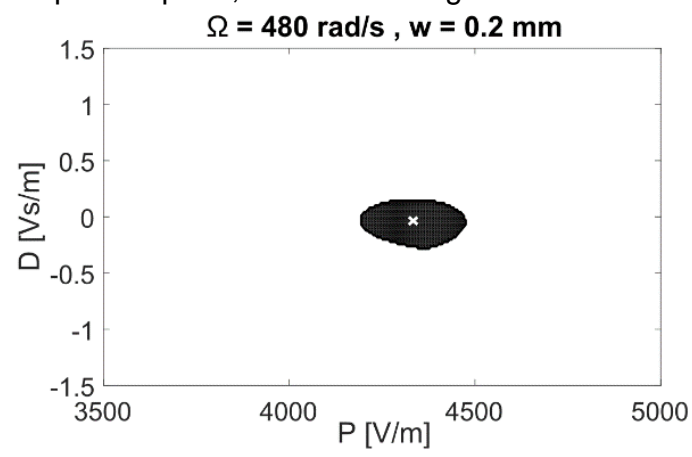

Fig. 6: Stable PD control parameters.

\subsection{PDA12 approach}

The previously discussed active PD control can be further improved with the application the of the $A 1$ and $A 2$ parameters into the control loop. Thus adequate $A 12$ parameters must be found for the previously selected stable PD parameters. The result of this search can be seen in Fig. 7. The search was yet again carried out in the $w-\Omega$ combination seen on Fig. 5 and with the PD parameters seen on Fig. 6. 


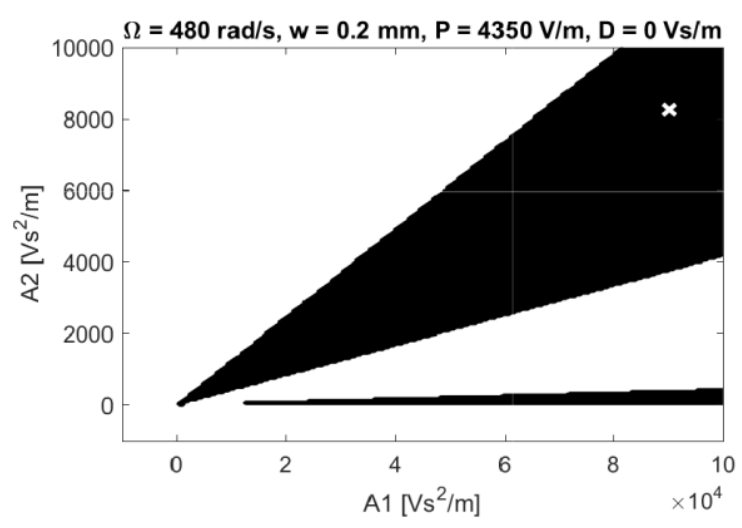

Fig. 7: Stable A12 control parameters.

As in the case of PD control development one stable point of operation is not sufficient. Yet again an arbitrarily chosen A1-A2 combination was selected and tested on the whole $w-\Omega$ stability map. Its results can be seen on Fig. 8 . Here it is clear, that due to the introduction of the $A 12$ parameters, the effect of the active enhancement became even more significant. The minimums of the lobes dropped even lower, and the maximum of the first lobe was perfectly aligned with the $w-\Omega$ combination chosen on Fig 5 .

Thus it is proven, that with the help of PDA12 control and the introduction of virtual masses, the lobes on the stability map can be moved in accordance with the used spindle speed, and the material removal can always be done in a "sweet spot", with high stability and material removal rate.

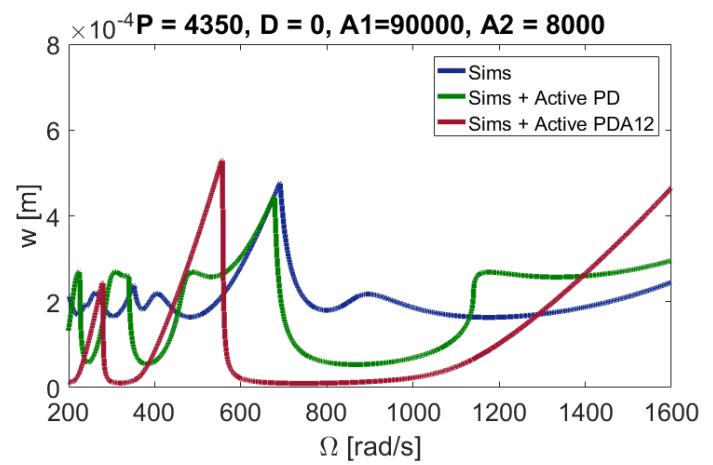

Fig. 8: The effect of PD and PDA12 control on the boring process.

\section{SUMMARY}

A novel method for boring bar vibration attenuation was presented and analyzed. A simplified linearized model of the cutting and nonlinear force system was formulated.

With the help of this created model, an ideal control strategy was developed for the applied nonlinear control force, and its effectiveness was tested through numerical simulations carried out via Linear Multistep method.

It was found that given the right control strategy, and the appropriate control parameters, the active enhancement of the damper applied onto the boring bar yields favorable results, and can significantly improve the stability, effectiveness and productivity of the machining process.

\section{ACKNOWLEDGMENTS}

This research was supported by the Hungarian Government in the framework of NKFI FK124361 research project, ÚNKP Bolyai+ (ÚNKP-18-4-BME-193) and EUROSTARS FORTH E!12998.

\section{REFERENCES}

[Abele 2016] Abele, E.; Haydn, M.; Grosch, T. : Adaptronic approach for modular long projecting boring tools. CIRP Annals 65.1 (2016): 393-396.

[Åkesson 2006] Åkesson, H. ; Smirnova, T. ; Håkansson, L. ; Claesson, I. ; Sigfridsson, A. ; Svensson, T. ; Lagö, T. : A First Prototype of an Active Boring Bar Tested in Industry. In: Thirteenth International Congress on Sound and Vibration (2006)

[Chen 2014] Chen, F.; Lu, X. ; Altintas, Y. : A novel magnetic actuator design for active damping of machining tools. In: International Journal of Machine Tools and Manufacture 85 (2014), p. 58 - 69

[Chiu 1997] Chiu, W.; Chan, K.: Design and testing of piezoelectric actuator-controlled boring bar for active compensation of cutting force induced errors. In: International Journal of Production Economics 51 (1997), Nr. 1-2, p. $135-148$

[Den Hartog 1934] Den Hartog, J.P.; "Mechanical Vibrations"; In: McGraw-Hill Book. Company, New York and London, 1934

[Engelborghs 2002] Engelborghs, K., \& Roose, D. On Stability of LMS Methods and Characteristic Roots of Delay Differential Equations. SIAM Journal on Numerical Analysis, 2002 . 40(2), 629-650. doi:10.1137/s003614290037472x

[Iserles 1996] Iserles, Arieh (1996), A First Course in the Numerical Analysis of Differential Equations, Cambridge University Press, ISBN 978-0-521-55655-2.

[Mei 2005] Mei, C. (2005). Active regenerative chatter suppression during boring manufacturing process. Robotics and Computer-Integrated Manufacturing, 21(2), 153-158. doi:10.1016/j.rcim.2004.07.011

[Marui 1988] Marui, E., Kato, S., Hashimoto, M., \&

Yamada, T. (1988). The Mechanism of Chatter Vibration in a Spindle-Workpiece System: Part 2-Characteristics of Dynamic Cutting Force and Vibration Energy. Journal of Engineering for Industry, 110(3), 242. doi:10.1115/1.3187876

[Munoa 2015] Jokin Munoa, Zoltan Dombovari, Alexander Iglesias, Gabor Stepan. Tuneable mass dampers with variable stiffness for chatter suppression. 4th International Conference on Virtual Machining Process Technology (VMPT 2015), CIRP, Jun 2015, Vancouver, Canada. 〈hal01338634>

[Munoa 2016] Munoa, J., Beudaert, X., Dombovari, Z., Altintas, Y., Budak, E., Brecher, C., \& Stepan, G. (2016). Chatter suppression techniques in metal cutting. CIRP Annals, 65(2), 785-808. doi:10.1016/j.cirp.2016.06.004 [Redmond 1999] Redmond, J. M. ; Barney, P. S. ; Parker, G. G. ; Smith, D. A.: Vibration damping method and apparatus. 1999. US patent 5,913,955

[Sims 2007] Sims, N. D. Vibration absorbers for chatter suppression: A new analytical tuning methodology. Journal of Sound and Vibration, 2007. 301(3-5), 592-607. doi:10.1016/j.jsv.2006.10.020

[Tanaka 1994] Tanaka, H. ; Obata, F. ; Matsubara, T. ; Mizumoto, H. : Active Chatter Suppression of Slender Boring Bar Using Piezoelectric Actuators. In: JSME International Journal Series C - Dynamics Control Robotics Design and Manufacturing 37 (1994), Nr. 3, p. $601-606$

EDITOR'S NOTICE: The paper does not present a full description of the actual realization the active boring bar. This was requested by reviewers during the review process. Authors are not allowed to present the detailed realization 
of the force actuation due to competitive legal protection. Thus, simplified model is used only. Since the paper presents valuable control strategy of the nonlinear active damper, the paper was accepted in this final version. 